\title{
EVOLVIMENTO DE DIÓXIDO DE CARBONO E MINERALIZAÇÃO DE NITROGÊNIO EM LATOSSOLO VERMELHO-ESCURO COM DIFERENTES MANEJOS'
}

\author{
TERESA CRISTINA LARA LANZA DE SÁ E MELO MARQUES², CARLOS ALBERTO VASCONCELLOS ${ }^{3}$,

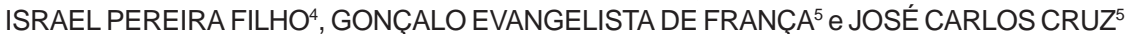

\begin{abstract}
RESUMO - Este trabalho foi elaborado com o objetivo de verificar o efeito das palhas de milho e lab-lab, dos tipos de manejos do solo e da presença de uma fonte externa de $\mathrm{N}$, no evolvimento de $\mathrm{CO}_{2}$ e na mineralização de N. Usou-se um Latossolo Vermelho-Escuro distrófico textura argilosa fase cerrado, da região de Sete Lagoas (MG), com histórico de uso envolvendo cinco anos sob plantio direto e plantio convencional com arado de disco e arado de aiveca. Amostras de solo $(0-20 \mathrm{~cm})$ foram incubadas a $25^{\circ} \mathrm{C}$ durante 55 dias, na presença e na ausência de palhada residual e de fonte externa de $\mathrm{N}$ aplicada no início e 25 dias após. De modo geral, a palhada de milho apresentou maior taxa de $\mathrm{CO}_{2}$ evolvido do que o lablab (935 e $764 \mu \mathrm{g} \mathrm{CO}_{2} \mathrm{~g}^{-1}$ solo, respectivamente). O tipo de palhada residual influenciou mais o evolvimento de $\mathrm{CO}_{2}$ do que os manejos de solo. A adição de $\mathrm{N}$ aumenta a taxa de $\mathrm{CO}_{2}$ evolvida, apenas quando $\mathrm{N}$ é aplicado no início do período de incubação. A disponibilidade de $\mathrm{N}$ e o tempo de imobilização são alterados pela relação $\mathrm{C} / \mathrm{N}$ da palhada incorporada e pelo preparo do solo.
\end{abstract}

Termos para indexação: palhada residual, imobilização, adubação nitrogenada.

\section{EVOLVED CARBON DIOXIDE AND NITROGEN MINERALIZATION IN A DARK-RED LATOSOL WITH DIFFERENT MANAGEMENTS}

\begin{abstract}
The objective of this work was to verify the influence of different types of straw (maize and lablab) and soil management on evolving $\mathrm{CO}_{2}$ and $\mathrm{N}$ mineralization. A dystrophic clayey Dark-Red Latosol under Cerrado from the region of Sete Lagoas, MG, Brazil, was cultivated during five years with no-tillage, disc-plow and moldboard-plow. Soil samples $(0-20 \mathrm{~cm})$ were incubated at $25^{\circ} \mathrm{C}$ during 55 days, with and without $\mathrm{N}$ fertilizer applied at the beginning and 25 days afterwards. As a general rule, the maize straw had higher rates of evolved $\mathrm{CO}_{2}$ than the lablab ( 935 e $764 \mu \mathrm{g} \mathrm{CO}_{2} \mathrm{~g}^{-1}$ soil, respectively). The type of straw influenced the amount of $\mathrm{CO}_{2}$ evolved more than the type of soil management did. Nitrogen addition increases the rate of $\mathrm{CO}_{2}$ evolved only when applied at the beginning of the incubation period. The $\mathrm{N}$ availability and the duration of the immobilization are modified by the $\mathrm{C} / \mathrm{N}$ ratio and by the type of soil management.
\end{abstract}

Index terms: mulching straw, immobilization, $\mathrm{N}$ fertilization.

${ }^{1}$ Aceito para publicação em 11 de março de 1999. Apresentado no XXV Congresso Brasileiro de Ciência do Solo, Viçosa, MG, 1995.

${ }^{2}$ Eng. Agrôn., M.Sc., 149-11 Arnold Drive, West Lafayette, IN, 47906-3339, USA. E-mail: tmarques@expert.cc.purdue.edu

${ }^{3}$ Eng. Agrôn., D.Sc., Embrapa-Centro Nacional de Pesquisa de Milho e Sorgo (CNPMS), Caixa Postal 151, CEP 35701-970 Sete Lagoas, MG. E-mail: carlos@cnpms.embrapa.br

${ }^{4}$ Eng. Agrôn., M.Sc., Embrapa-CNPMS

${ }^{5}$ Eng. Agrôn., Ph.D., Embrapa-CNPMS

\section{INTRODUÇÃO}

Ao se adicionar material orgânico ao solo, este é mineralizado pela ação dos microrganismos, que liberaram os elementos minerais contidos neste material (Alexander, 1977). A estimativa da atividade microbiológica pode ser feita pela taxa de respiração ou por diversas outras metodologias envolvendo a identificação da flora microbiana. A atividade 
metabólica específica é expressa como atividade de respiração própria, a qual representa a produção de $\mathrm{CO}_{2}$ por unidade de biomassa e unidade de tempo (Santruckova \& Straskraba, 1991). A respiração do solo está relacionada com a disponibilidade de $\mathrm{C}$ na biomassa, e é, geralmente, maior na superfície de solos sob plantio direto, por causa da melhor atividade biológica (Carter, 1986; Follett \& Schimel, 1989). Uma alta taxa respiratória indicará maior atividade microbiana e uma decomposição mais rápida do material orgânico do solo, com conseqüente liberação de nutrientes para as plantas. Este fenômeno de liberação de nutrientes ao solo é chamado mineralização, e o de sua retenção em forma orgânica na biomassa microbiana, imobilização (Bartholomew, 1965). Há, contudo, a possibilidade de a biomassa do solo crescer e atuar na decomposição da matéria orgânica nativa, diminuindo sua fertilidade, por ser um dos fatores contribuintes do que se denomina priming effect.

A quantidade de $\mathrm{N}$ no material orgânico determinará a velocidade da sua decomposição. Muitos estudos têm mostrado que resíduos de baixa relação $\mathrm{C} / \mathrm{N}$ decompõem-se mais rápido que os de relação $\mathrm{C} / \mathrm{N}$ alta. A baixa velocidade de decomposição dos resíduos de alta relação $\mathrm{C} / \mathrm{N}$ tem sido atribuída à deficiência de $\mathrm{N}$ e à presença de constituintes recalcitrantes (Alexander, 1977). Segundo Swift et al. (1979), os decompositores possuem menor relação $\mathrm{C} / \mathrm{N}$ do que as fontes por eles exploradas, sendo então $\mathrm{N}$ reconhecido como um fator limitante para o crescimento e atividade microbiana durante a decomposição de resíduos de plantas. Dessa forma, muitos estudos sustentam evidências de que a adição de $\mathrm{N}$ estimula a decomposição de resíduos incorporados ao solo. Reinertsen et al. (1984) estudaram o evolvimento do $\mathrm{CO}_{2}$ da palha de trigo, variando a relação $\mathrm{C} / \mathrm{N}$ mediante a adição de $\mathrm{N}$ por fertilizantes. $\mathrm{A}$ adição de $\mathrm{N}$ aumentou a liberação de $\mathrm{CO}_{2}$ na presença de palha de trigo. Contudo, quando na presença da palhada de leguminosa, este aumento foi menor, principalmente no estádio inicial de decomposição. Neely et al. (1991) verificaram que, para os resíduos estudados, a relação $\mathrm{C} / \mathrm{N}$ correlacionou-se positiva e significativamente com a respiração total.

O manejo do solo e da cultura, portanto, influencia a atividade da flora microbiana do solo. Follett \&
Schimel (1989) verificaram que há diminuição da capacidade do solo em imobilizar e conservar N mineral com o aumento dos tratos culturais. Esses mesmos autores observaram diminuição da quantidade de respiração microbiana na seguinte ordem: solo gramado, solo sob pousio, solo com restos de cultura e solo arado.

Dentro de um mesmo solo, o preparo mecânico, o manejo da palhada residual e do fertilizante nitrogenado, podem alterar o comportamento de seus componentes biológicos e, conseqüentemente, a utilização dos nutrientes pelas plantas cultivadas.

O objetivo deste trabalho foi verificar a influência do resíduo vegetal, do tipo de preparo de solo e da presença de fonte externa de $\mathrm{N}$ aplicada em duas épocas durante o período de incubação, sobre a taxa de evolvimento de $\mathrm{CO}_{2}$ e a mineralização do $\mathrm{N}$.

\section{MATERIAL E MÉTODOS}

$\mathrm{O}$ experimento, do qual se originaram as amostras de solo, foi conduzido na Embrapa-Centro Nacional de Pesquisa de Milho e Sorgo (CNPMS), em Sete Lagoas, MG, num Latossolo Vermelho-Escuro distrófico, textura argilosa, relevo suave ondulado, fase cerrado. $\mathrm{O}$ histórico de uso envolveu cinco anos, com diferentes preparos de solo: (i) plantio direto, (ii) preparo com arado de disco e grade niveladora e (iii) com arado de aiveca e grade niveladora.

$\mathrm{O}$ experimento foi composto por tratamentos obtidos por fatorial completo entre três diferentes preparos de solo; três tipos de resíduo cultural: sem palha, palha de lab-lab (Dolichos lablab) e palha de milho (Zea mays); duas épocas de aplicação da adubação nitrogenada: no início da incubação e 25 dias após o início da incubação. Em todos os tratamentos foram feitas duas repetições.

A palhada de milho foi obtida separando-se os nós e os entre-nós do colmo e folhas, moendo-as e passando-as em peneiras de $1 \mathrm{~mm}$. Procurando-se manter a proporção dessas diferentes partes, pesou-se $0,4 \mathrm{~g}$ do nó, 2,4 g do entre-nó e 0,2 g de folha por kilograma de solo secado ao ar. A palha de lab-lab foi uniformemente moída, peneirada (1 mm), e incorporada ao solo, na proporção de $3 \mathrm{~g} \mathrm{~kg}^{-1} \mathrm{de}$ solo. A palha de lab-lab e a palha de milho possuíam, respectivamente, relação $\mathrm{C} / \mathrm{N}$ de 28 e de 35 , com 10,5 e $6 \mathrm{~g} \mathrm{~kg}^{-1}$ de N. Amostras da camada de 0-20 cm foram destorroadas e passadas em peneiras de $2 \mathrm{~mm}$. Até a instalação do ensaio, o solo foi mantido em local sombreado, com umidade entre 200 e $300 \mathrm{~g} \mathrm{~L}^{-1}$ do volume total de poros (VTP). A determinação dos parâmetros indicadores da fertilidade inicial das amostras, efetuada segundo 
metodologia descrita por Embrapa (1997), apresentou $\mathrm{pH}$ em $\mathrm{H}_{2} \mathrm{O}$ de 6,1 no tocante aos manejos com arado de aiveca e plantio direto, e 6,4 no manejo com arado de disco. As concentrações de $\mathrm{P}$ nos manejos com aiveca, disco e plantio direto foram 23,39 e $50 \mu \mathrm{g} \mathrm{g}^{-1}$ solo e a CTC efetiva encontrada para esses manejos foram 7,6, 8,6 e $8,1 \mathrm{cmol} \mathrm{kg}^{-1}$, respectivamente. O teor de matéria orgânica foi de $4 \mathrm{~g} \mathrm{~kg}^{-1} \mathrm{em}$ todos os manejos de solo estudados.

Em cada tratamento foram pesados $20 \mathrm{~g}$ de solo secado ao ar, incorporando-se os resíduos culturais. Fósforo, na forma de superfosfato simples, foi aplicado na quantidade de $50 \mathrm{~kg} \mathrm{ha}^{-1}$ de $\mathrm{P}_{2} \mathrm{O}_{5}$. A irrigação foi feita para atingir $400 \mathrm{~g} \mathrm{~L}^{-1}$ do VTP. A umidade foi mantida constante, repondo-se a água através do controle de peso. Como fonte de $\mathrm{N}$, utilizou-se $\mathrm{NH}_{4}\left(\mathrm{SO}_{4}\right)_{2}$, aplicando-se $15 \mu \mathrm{g} \mathrm{N} \mathrm{g}{ }^{-1}$ solo, juntamente com a água de irrigação. Aos 25 dias após o início do experimento, adicionou-se $\mathrm{N}$ nos tratamentos que recebiam $\mathrm{N}$ em cobertura. Cada tratamento foi mantido em frascos de vidro hermeticamente fechados, com capacidade para $5 \mathrm{~L}$, em temperatura constante de $25^{\circ} \mathrm{C}$, para amostragens nas épocas preestabelecidas.

No interior de cada frasco foram colocados frascos menores contendo $20 \mathrm{~mL}$ de solução $\mathrm{NaOH} 1 \mathrm{~N}$, para avaliação do $\mathrm{CO}_{2}$ evolvido, como especificado por Jenkinson \& Powlson (1976). A primeira coleta (época) foi feita aos cinco dias, e depois, a cada dez dias efetuava-se nova coleta. A cada coleta, um dos frascos era retirado, para a determinação do nitrato e do amônio. A extração do $\mathrm{N}$ mineral foi feita com $\mathrm{KCl} 2 \mathrm{~N}$, numa proporção de $3: 1$, agitando-se por 15 minutos, seguindo-se a filtração em papel. Posteriormente, o $\mathrm{NH}_{4}^{+}$e o $\mathrm{NO}_{3}^{-}$foram determinados segundo metodologia de Bremner \& Keeney (1966).

Os dados foram submetidos à análise da variância e a testes de médias, considerando-se 5\% como nível de significância. O teste de médias utilizado foi o de Duncan.

\section{RESULTADOS E DISCUSSÃO}

\section{$\mathrm{CO}_{2}$ evolvido com $\mathrm{N}$ adicionado no início da incubação}

$\mathrm{Na}$ Fig. 1 encontra-se a produção diária de $\mathrm{CO}_{2} \mathrm{e}$ a produção acumulada do $\mathrm{CO}_{2}$ evolvido em todos os tipos de palhadas e manejos de solo utilizados, em função do período de incubação. A adição de $\mathrm{N}$ na ausência de palhada residual não provocou grandes alterações na produção de $\mathrm{CO}_{2}$, o qual manteve os menores valores diários ao longo do período estudado (Fig. 1a), provavelmente devido à limitação de substratos energéticos no solo. Nos tratamentos que receberam palhadas residuais, a quantidade de $\mathrm{CO}_{2}$ evolvido foi maior naqueles com adição de palha de milho. Contudo, não houve diferença quanto ao manejo do solo. Diferenças foram observadas apenas no nível inicial do $\mathrm{CO}_{2}$ evolvido diariamente, o que demonstra haver diferenças entre manejos de solo no que se refere ao estado inicial da matéria orgânica. Depois do $15^{\circ}$ dia de incubação, a evolução diária de $\mathrm{CO}_{2}$ nos tratamentos com plantio direto, tanto para a palha de milho quanto para a palha de lab-lab, foi igual. Provavelmente, ao se adicionar $\mathrm{N}$ no início do período de incubação colocou o tratamento com palhada de milho (maior relação $\mathrm{C} / \mathrm{N}$ ) nas mesmas condições do tratamento com palha de lab-lab (menor relação C/N).

Verificou-se maior produção acumulada de $\mathrm{CO}_{2}$ nos tratamentos que receberam palhada residual de milho (Fig. 1b). Este resultado pode ser devido à moagem do material que foi incorporado ao solo. Estando o material moído, o acesso dos microrganismos do solo ao substrato é facilitado pelo aumento da área superficial e pela redução na proteção dos compostos ricos em C (Swift et al., 1979). Bremer et al. (1991) observaram que a moagem não afetou a evolução do $\mathrm{CO}_{2}$ do tratamento usando adubação verde com lentilha, porém aumentou a evolução de $\mathrm{CO}_{2}$ do tratamento com palha de trigo. Este aumento foi atribuído ao maior acesso ao $\mathrm{C}$ moderadamente disponível ao ataque microbiano (p.e. celulose, hemicelulose).

Nos tratamentos com palha de lab-lab, a produção acumulada de $\mathrm{CO}_{2}$ foi maior no plantio direto (787 $\mu \mathrm{g} \mathrm{CO}_{2} \mathrm{~g}^{-1}$ solo) do que no manejo com arado de disco e aiveca (741 e $751 \mu \mathrm{g} \mathrm{CO}_{2} \mathrm{~g}^{-1}$ solo, respectivamente), os quais mostraram pequena diferença entre si. O fato de o resíduo cultural permanecer na superfície do solo quando é utilizado o plantio direto, favorecendo o desenvolvimento de uma biomassa mais ativa nas camadas superiores, contribuiu para maiores taxas de evolvimento de $\mathrm{CO}_{2}$. Pode-se inferir também sobre a possibilidade da atividade de microrganismos externos ao solo distribuídos sobre o tecido vegetal que, quando incorporados, ajudam na decomposição dos resíduos vegetais. Alvarez et al. (1995a) verificaram que a respiração do solo foi maior na superfície $(0-5 \mathrm{~cm})$ em condições de plantio 

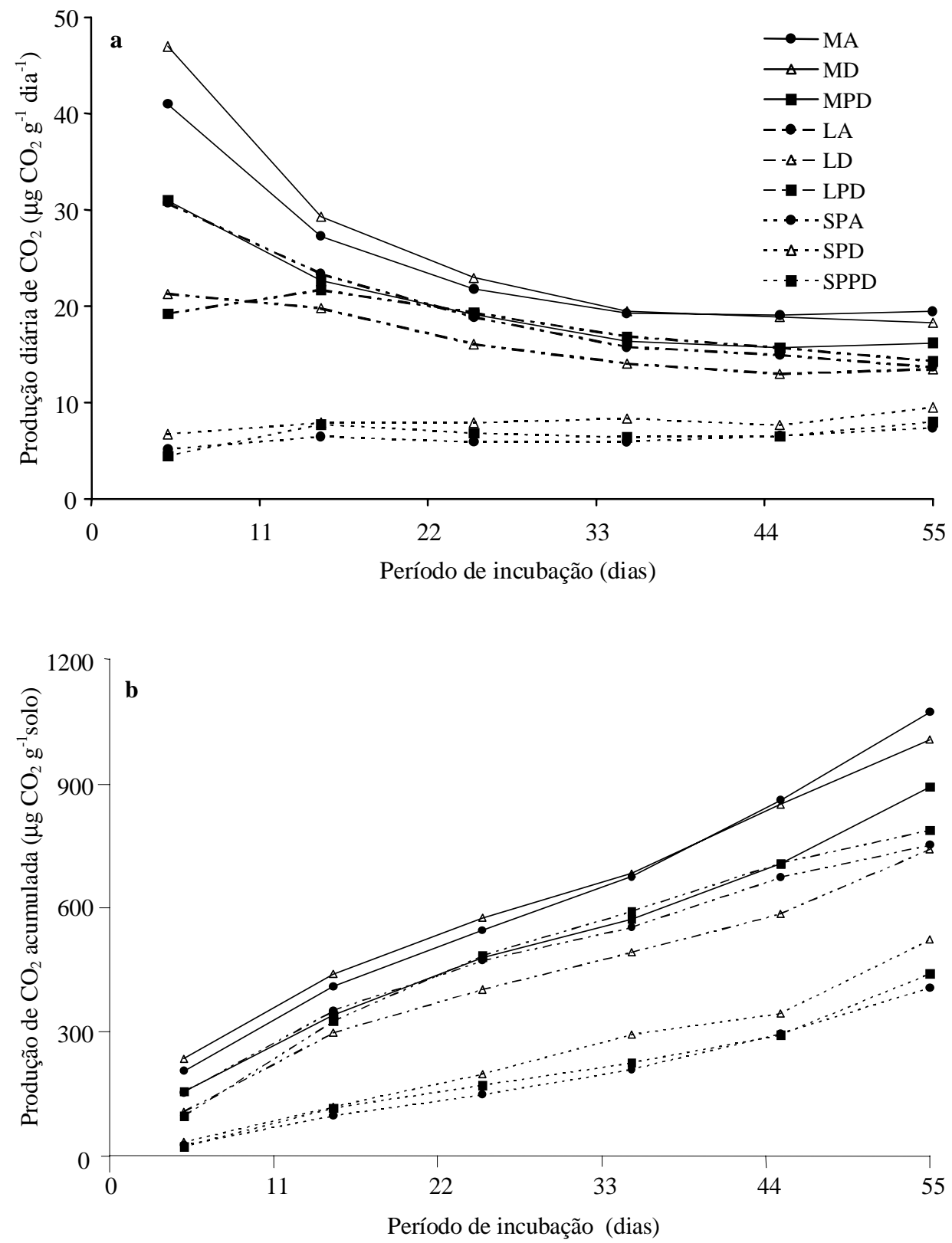

FIG. 1. Efeito do resíduo cultural sobre produção diária de $\mathrm{CO}_{2}$ (a) e sobre a produção acumulada de $\mathrm{CO}_{2}$ evolvido (b) em LEd submetido a diferentes manejos de solo, com $\mathrm{N}$ aplicado no início da incubação. MA: palhada de milho, aiveca; MD: palhada de milho, disco; MPD: palhada de milho, plantio direto; LA: palhada de lab-lab, aiveca; LD: palhada de lab-lab, disco; LPD: palhada de lab-lab, plantio direto; SPA: sem palhada, aiveca; SPD: sem palhada, disco; SPPD: sem palhada, plantio direto. 
direto que em solo arado, e que na profundidade de $10-15 \mathrm{~cm}$ o oposto foi verificado. Hendrix et al. (1988) e Prior et al. (1997) também encontraram maior evolução de $\mathrm{CO}_{2}$ no preparo de solo com plantio direto e utilizando palhadas residuais com baixa relação $\mathrm{C} / \mathrm{N}$. Contudo, Alvarez et al. (1995b) verificaram maior taxa de $\mathrm{CO}_{2}$ evolvido no preparo de solo convencional que no preparo de solo usando o cultivo mínimo.

Quando se comparam as duas palhadas (lab-lab e milho), observa-se que as taxas de evolvimento no plantio direto foram as mesmas, enquanto os preparos de solo com aiveca e disco obtiveram maiores valores na palha de milho. Provavelmente, nestes tratamentos os microrganismos são menos selecionados, em função da constante movimentação do solo e menos influenciados pela relação $\mathrm{C} / \mathrm{N}$ do resíduo cultural. Chan et al. (1992) verificaram que áreas sob aração e gradagem tiveram maior evolvimento de $\mathrm{CO}_{2}$.

A menor evolução de $\mathrm{CO}_{2}$ nos preparos de solo com aiveca e disco nos tratamentos com palha de lab-lab pode também ser explicada pelo fato de a energia introduzida ter sido incorporada à biomassa do solo. Sampaio \& Salcedo (1982) encontraram menor evolução de $\mathrm{CO}_{2}$ quando houve aumento da biomassa com adição de $\mathrm{N}$ e $0,03 \%$ de palha de milho, o que concorda com a hipótese de incorporação de $\mathrm{C}$ à biomassa.

\section{$\mathrm{CO}_{2}$ evolvido na presença de $\mathrm{N}$ adicionado aos 25} dias após início da incubação

O comportamento dos tratamentos com adição de N 25 dias após a incubação encontra-se na Fig. 2. Como observado quanto ao $\mathrm{N}$ adicionado no início do período de incubação, a adição de $\mathrm{N}$ aos 25 dias nos tratamentos sem palhada residual pouco alterou a quantidade de $\mathrm{CO}_{2}$ evolvido, ou seja, a ausência de uma fonte energética limitou o desenvolvimento dos microrganismos, não importando a época de adição de $\mathrm{N}$. No entanto, a produção de $\mathrm{CO}_{2}$ no tratamento com arado de disco mostrou-se superior ao plantio direto e ao arado de aiveca (365, 234 e $189 \mu \mathrm{g} \mathrm{CO}_{2} \mathrm{~g}^{-1}$ solo, respectivamente), na ausência de palhada.

$\mathrm{A}$ adição de $\mathrm{N}$ aos 25 dias de incubação não aumentou a quantidade diária de $\mathrm{CO}_{2}$ evolvida, nos tratamentos com palha de lab-lab e palha de milho. A taxa de evolução diária de $\mathrm{CO}_{2}$, no quinto dia de incubação, foi inferior nos tratamentos que receberam $\mathrm{N}$ aos 25 dias (Fig. 2a) do que naqueles com $\mathrm{N}$ adicionado no início do período (Fig. 1a). Knapp et al. (1983) somente observaram aumento na evolução de $\mathrm{CO}_{2}$ quando $\mathrm{N}$ foi adicionado imediatamente à palha de milho. Quando esses autores adicionaram a mesma quantidade de $\mathrm{N}$ dez dias depois, não houve estimulação na evolução de $\mathrm{CO}_{2}$. A resposta limitada à adição de $\mathrm{N}$ pode estar relacionada à menor relação $\mathrm{C} / \mathrm{N}$ dos constituintes solúveis, do que do resíduo de plantas como um todo, e também à capacidade dos microrganismos de se adaptarem a baixas condições de N disponível (Levi \& Cowling, 1969).

Verifica-se que, dentro de todos os tipos de palhadas estudadas, com $\mathrm{N}$ adicionado aos 25 dias, o $\mathrm{CO}_{2}$ diário evolvido no quinto dia de incubação foi maior no manejo de solo com arado de aiveca, seguido pelo arado de disco, e, por último, o plantio direto (Fig. 2a). Também pode-se observar, em todas as palhadas estudadas, maior decréscimo na taxa diária de $\mathrm{CO}_{2}$ evolvido em relação ao arado de aiveca, e menor decréscimo no plantio direto. Apesar da profundidade de amostragem dos solos $(0-20 \mathrm{~cm})$, os tratamentos com plantio direto ainda mostraram melhor equilíbrio de sua microbiota, alterando menos a quantidade diária de $\mathrm{CO}_{2}$ evolvido.

Novamente a palha de milho apresentou maior taxa de evolução de $\mathrm{CO}_{2}$ (plantio direto, 1059; disco, 899; aiveca, $685 \mu \mathrm{g} \mathrm{CO} \mathrm{CO}_{2} \mathrm{~g}^{-1}$ solo) do que a palha de lab-lab (disco, 837; aiveca, 767; plantio direto, $703 \mu \mathrm{g} \mathrm{CO}_{2} \mathrm{~g}^{-1}$ solo), com exceção do tratamento com palha de milho e arado de aiveca (Fig. 2b). A produção acumulada de $\mathrm{CO}_{2}$ foi maior no plantio direto, usando palha de milho. Provavelmente, a microbiota mais ativa no plantio direto e a incorporação da palha de milho moída, que facilitou o acesso dos microrganismos ao $\mathrm{C}$ da planta, favoreceram a maior evolução de $\mathrm{CO}_{2}$. Nos tratamentos com palha de lab-lab, a adição de $\mathrm{N}$ aos 25 dias de incubação pouco diferiu entre os manejos do solo estudados.

A comparação entre as duas fontes energéticas utilizadas permite observar que o comportamento dos tratamentos com arado de aiveca e arado de disco mantiveram-se constantes, havendo apenas uma variação da quantidade de $\mathrm{CO}_{2}$ evolvido no período 
final, implicando uma microbiota menos exigente em $\mathrm{N}$ (Fig. 2b). O plantio direto mostrou maior resposta ao tipo de palhada empregada. Portanto, o principal fator a influenciar estas diferenças deve estar relacionado com a relação $\mathrm{C} / \mathrm{N}$ das fontes energéticas adi- cionadas. Neely et al. (1991) verificaram que em todos os resíduos de plantas, a relação $\mathrm{C} / \mathrm{N}$ foi o principal fator na determinação da respiração total. $\mathrm{Na}$ presença de lab-lab, a flora microbiana existente não foi limitada por fonte externa de N, pelo fato de pos-
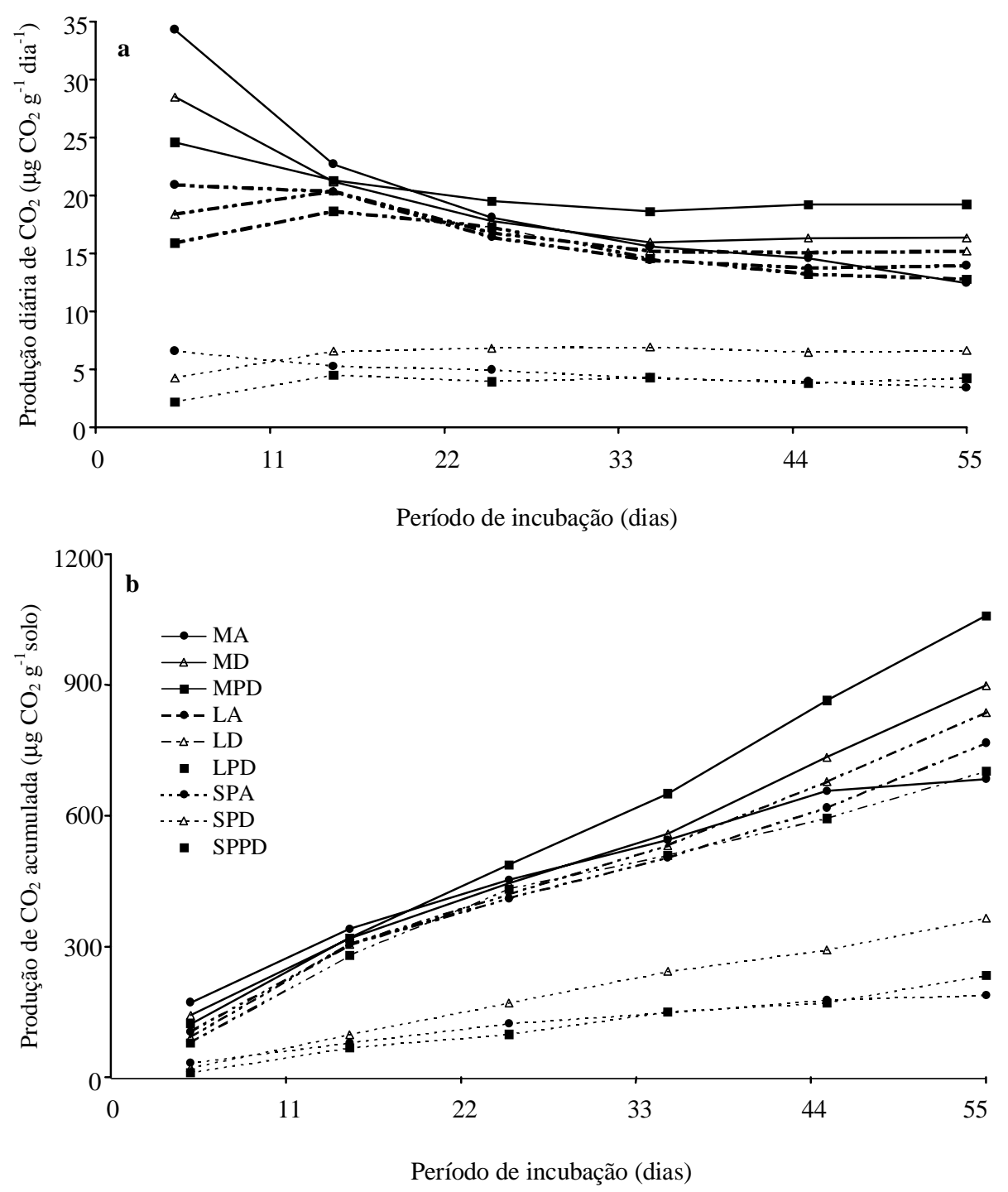

FIG. 2. Efeito do resíduo cultural sobre produção diária de $\mathrm{CO}_{2}$ (a) e sobre produção acumulada de $\mathrm{CO}_{2}$ evolvido (b) em LEd submetido a diferentes manejos de solo, com $\mathbf{N}$ aplicado aos 25 dias de incubação. MA: palhada de milho, aiveca; MD: palhada de milho, disco; MPD: palhada de milho, plantio direto; LA: palhada de lab-lab, aiveca; LD: palhada de lab-lab, disco; LPD: palhada de lab-lab, plantio direto; SPA: sem palhada, aiveca; SPD: sem palhada, disco; SPPD: sem palhada, plantio direto. 
suir uma quantidade suficiente àquela requerida pelos decompositores. Reinertsen et al. (1984) verificaram que as adições de $\mathrm{N}$ não afetaram a evolução do $\mathrm{CO}_{2}$ da palha de trigo com a relação $\mathrm{C} / \mathrm{N}$ de 36 . Houve, contudo, aumento da evolução de $\mathrm{CO}_{2}$ da palha de trigo com a relação $\mathrm{C} / \mathrm{N}$ de 54 e 238 , o que indica que a ausência de uma fonte externa de $\mathrm{N}$ pode não limitar a taxa de decomposição de resíduos de plantas que tenham uma relação $\mathrm{C} / \mathrm{N}$ menor ou igual a 40 .

\section{Mineralização de N}

A análise de variância dos teores de $\mathrm{N}$ amoniacal das amostras, na maioria das épocas estudadas, mostrou efeito não-significativo em todas as fontes de variações: preparo do solo, palha, $\mathrm{N}$ no plantio ou em cobertura, e suas respectivas interações.

Os altos coeficientes de variação indicaram diferentes precisões entre épocas, ou seja, houve influência de fatores não controlados. Provavelmente, a decomposição da palha por etapas, associada à solubilidade de compostos orgânicos, pode explicar esta variabilidade encontrada (C.V. de $22 \%$ a $54 \%$ ).

A Tabela 1 mostra as concentraçãos de $\mathrm{N}$ inorgânico em todas as palhadas e manejos de solo estudados nos diferentes períodos de incubação. Nos primeiros dias de incubação, devido a adição de uma fonte energética, houve uma queda no teor de $\mathrm{N}$ amoniacal, mostrando o efeito da imobilização do N pela microbiota do solo. Dos 15 aos 25 dias da incubação, houve mineralização do $\mathrm{N}$ observada pelo aumento do teor de $\mathrm{N}$ amoniacal. Algum fator, como a presença de substância liberada pela decomposição do resíduo, atividade e/ou especificidade da biomassa, deve ter favorecido a amonificação, pois observou-se um baixo efeito dos tratamentos sobre o teor de nitrato nesse período. Azam et al. (1995) sugeriram que o $\mathrm{N}$ amoniacal pode ser imobilizado mais rapidamente do que $\mathrm{N}$ nítrico, por causa da diferença qualitativa do tipo de população microbiana que desenvolve. Quando foi feita a adubação nitrogenada de cobertura aos 25 dias, verificou-se um novo período de imobilização, seguindo-se a mineralização posterior aos 45 dias de incubação. Este fato pode indicar a necessidade de $\mathrm{N}$ no sistema para o turnover dos microrganismos na ciclagem do $\mathrm{N}$ e na decomposição dos resíduos incorporados, e também necessidades diferenciais das adubações de cobertura com $\mathrm{N}$.

Maiores valores de $\mathrm{N}$ nítrico (Tabela 1) foram obtidos nos tratamentos com palha de lab-lab (máximo de $50 \mu \mathrm{g} \mathrm{N}-\mathrm{NO}_{3}^{-} \mathrm{g}^{-1}$ solo) do que com palha de milho (máximo de $25 \mu \mathrm{g} \mathrm{N}-\mathrm{NO}_{3}^{-} \mathrm{g}^{-1}$ solo), talvez por causa da menor relação $\mathrm{C} / \mathrm{N}$ da palha de lab-lab, fornecendo maior quantidade de $\mathrm{N}$ para o sistema. $\mathrm{O}$ menor teor de $\mathrm{N}$ nítrico no período de 5 a 25 dias de incubação, nos tratamentos com palha de lab-lab,

TABELA 1. Efeito de palhadas residuais sobre a concentração média de amônio e nitrato dos tratamentos com e sem adição de $\mathrm{N}$, em LEd submetido a diferentes manejos de solo ${ }^{1}$.

\begin{tabular}{|c|c|c|c|c|c|c|c|c|c|c|c|}
\hline \multirow[t]{3}{*}{ Tratamento } & \multicolumn{11}{|c|}{ Dias de incubação } \\
\hline & \multicolumn{2}{|r|}{5} & \multicolumn{2}{|c|}{15} & \multicolumn{2}{|c|}{25} & \multicolumn{2}{|c|}{35} & \multicolumn{2}{|c|}{45} & 55 \\
\hline & $\mathrm{NH}_{4}^{+}$ & $\mathrm{NO}_{3}^{-}$ & $\mathrm{NH}_{4}^{+}$ & $\mathrm{NO}_{3}^{-}$ & $\mathrm{NH}_{4}^{+}$ & $\mathrm{NO}_{3}^{-}$ & $\mathrm{NH}_{4}^{+}$ & $\mathrm{NO}_{3}^{-}$ & $\mathrm{NH}_{4}{ }^{+}$ & $\mathrm{NO}_{3}^{-}$ & $\mathrm{NH}_{4}{ }^{+} \mathrm{NO}_{3}{ }^{-}$ \\
\hline & & & & & & $---(11)$ & $\left.\mathrm{kg}^{-1}\right)$ & & & & \\
\hline Sem palha + aiveca & $4,0 \mathrm{a}$ & $13,7 \mathrm{ab}$ & $2,7 \mathrm{a}$ & $21,2 \mathrm{a}$ & $6,0 \mathrm{a}$ & $9,7 \mathrm{~b}$ & $4,6 \mathrm{a}$ & $59,3 \mathrm{a}$ & $2,0 \mathrm{a}$ & $37,4 \mathrm{a}$ & $4,3 a \quad 46,3 a$ \\
\hline Sem palha + disco & $3,8 \mathrm{a}$ & $13,9 \mathrm{ab}$ & $3,3 \mathrm{a}$ & $22,4 \mathrm{a}$ & $5,7 \mathrm{a}$ & $10,2 \mathrm{a}$ & $6,8 \mathrm{a}$ & $33,9 \mathrm{c}$ & $2,0 \mathrm{a}$ & $36,6 \mathrm{a}$ & $3,7 \mathrm{a} \quad 41,2 \mathrm{~b}$ \\
\hline Sem palha + pl. direto & $3,6 \mathrm{a}$ & $16,6 \mathrm{a}$ & $1,8 \mathrm{a}$ & $23,4 \mathrm{a}$ & $4,5 \mathrm{a}$ & $9,7 \mathrm{a}$ & $5,6 \mathrm{a}$ & $60,6 a$ & $3,3 \mathrm{a}$ & $41,1 \mathrm{a}$ & $3,5 \mathrm{a} \quad 30,9 \mathrm{c}$ \\
\hline Lab-lab + aiveca & $4,6 a$ & $4,0 \mathrm{de}$ & $2,5 \mathrm{a}$ & $5,5 b c$ & $6,3 a$ & $4,1 b$ & $4,1 \mathrm{a}$ & $35,6 c$ & $2,7 \mathrm{a}$ & $20,4 b$ & $5,0 \mathrm{a} \quad 26,3 \mathrm{cde}$ \\
\hline Lab-lab + disco & $5,0 \mathrm{a}$ & $6,8 \mathrm{~cd}$ & $2,2 \mathrm{a}$ & $4,9 b c$ & $6,5 \mathrm{a}$ & $3,2 b$ & $4,9 \mathrm{a}$ & $50,6 \mathrm{~b}$ & $2,2 \mathrm{a}$ & $18,4 \mathrm{bc}$ & $5,9 \mathrm{a} \quad 24,4 \mathrm{de}$ \\
\hline Lab-lab + pl. direto & $3,9 \mathrm{a}$ & $10,0 \mathrm{bc}$ & $2,0 \mathrm{a}$ & $9,6 \mathrm{~b}$ & $5,8 \mathrm{a}$ & $1,5 b$ & $6,3 \mathrm{a}$ & $20,6 \mathrm{~d}$ & $2,3 \mathrm{a}$ & $14,5 \mathrm{~cd}$ & $4,8 \mathrm{a} \quad 21,3 \mathrm{ef}$ \\
\hline Milho + aiveca & $3,4 \mathrm{a}$ & $1,1 \mathrm{e}$ & $2,2 \mathrm{a}$ & $1,5 \mathrm{c}$ & $6,5 a$ & $1,3 b$ & $5,9 \mathrm{a}$ & $14,3 \mathrm{ef}$ & $2,9 a$ & $15,6 \mathrm{bcd}$ & $7,7 \mathrm{a} \quad 16,4 \mathrm{f}$ \\
\hline Milho + disco & $4,4 \mathrm{a}$ & $0,6 \mathrm{e}$ & $2,6 a$ & $1,5 \mathrm{c}$ & $7,3 \mathrm{a}$ & $1,2 b$ & $4,7 \mathrm{a}$ & $9,7 \mathrm{f}$ & $2,9 \mathrm{a}$ & $17,4 b c$ & $8,1 \mathrm{a} \quad 18,5 \mathrm{f}$ \\
\hline Milho + pl. direto & $3,6 \mathrm{a}$ & $0,7 \mathrm{e}$ & $1,9 \mathrm{a}$ & $3,5 \mathrm{c}$ & $6,9 \mathrm{a}$ & $0,6 \mathrm{~b}$ & $7,6 \mathrm{a}$ & $15,2 \mathrm{e}$ & $2,0 \mathrm{a}$ & $10,9 \mathrm{~d}$ & $3,8 \mathrm{a} \quad 27,2 \mathrm{~cd}$ \\
\hline
\end{tabular}

${ }^{1}$ Nas colunas, números seguidos pela mesma letra são estatisticamente iguais entre si pelo teste de Duncan a $5 \%$ de probabilidade. 
pode ter sido ocasionado pela predominância da imobilização, uma vez que neste período o manejo do solo com o plantio direto apresentou valor inicial maior (10 $\mu \mathrm{g} \mathrm{N}-\mathrm{NO}_{3}^{-} \mathrm{g}^{-1}$ solo). Este maior valor de $\mathrm{N}$ encontrado no plantio direto pode ser devido ao maior fornecimento de $\mathrm{N}$ no solo por esse sistema. A partir dos 25 dias, o N imobilizado foi liberado, sendo observado um maior valor para o manejo de solo com arado de aiveca, seguindo-se o arado de disco e o plantio direto. $\mathrm{O}$ maior valor de $\mathrm{N}$ nítrico no emprego de aiveca pode ser devido ao priming effect, pois o revolvimento do solo, a uniformidade da incorporação e a profundidade de sua aração na incorporação de resíduos favorece o desenvolvimento de uma flora microbiana mais ativa (Follett \& Schimel, 1989).

Com a palha de milho, todos os manejos numa mesma época mostraram-se estatisticamente iguais, com exceção da última coleta, onde, no plantio direto, o teor de $\mathrm{N}$ nítrico foi maior do que nos outros dois manejos. Este maior valor pode ser causado pela existência de uma flora microbiana mais específica, pois observou-se menor valor do teor de $\mathrm{N}$ amoniacal nesta mesma época.

Observou-se que em todos os períodos de incubação, na ausência de palha, houve alta taxa de nitrificação (alto $\mathrm{N}$ nítrico), o que indica que na ausência de palhada, o processo de nitrificação foi maior do que o de amonificação. Aos 35 dias de incubação, o manejo com aiveca e o manejo com plantio direto apresentaram maior taxa de mineralização, alcançando valores de $60 \mu \mathrm{g} \mathrm{N}-\mathrm{NO}_{3}^{-} \mathrm{g}^{-1}$ solo. No preparo com arado de disco, o solo é homogenizado, favorecendo condições para o desenvolvimento microbiano na camada arável, sendo provável um turnover mais rápido, graças à maior superfície de contacto do solo com o material vegetal incorporado, o que justifica os resultados diferenciados deste manejo quando na ausência da aplicação de resíduo vegetal.

De modo geral, até aos 45 dias de incubação, quando diminuiu a concentração de $\mathrm{N}$ amoniacal, aumentou a de $\mathrm{N}$ nítrico. Após este período (de 45 a 55 dias de incubação), tanto no tocante ao $\mathrm{N}$ amoniacal quanto ao $\mathrm{N}$ nítrico, houve aumento de suas concentrações. É provável que os microrganismos já estejam mais em equilíbrio, ou seja, mais selecionados, graças à ausência da adição de C facilmente mineralizável.

\section{CONCLUSÕES}

1. A atividade dos microrganismos do solo, representados pelo teor de $\mathrm{CO}_{2}$ liberado e pela mineralização do N orgânico, é influenciada pela qualidade dos resíduos orgânicos adicionados e pelo tipo de preparo do solo.

2. A adição de $\mathrm{N}$ aumenta a taxa de $\mathrm{CO}_{2}$ evolvida, apenas quando o $\mathrm{N}$ é aplicado no início do período de incubação.

3. A disponibilidade do $\mathrm{N}$ e o tempo de imobilização após adição de resíduo cultural são alterados por causa da relação $\mathrm{C} / \mathrm{N}$ destes resíduos e do manejo de solo.

\section{AGRADECIMENTOS}

À Fundação de Amparo à Pesquisa do Estado de Minas Gerais (FAPEMIG), pelo financiamento deste projeto.

\section{REFERÊNCIAS}

ALEXANDER, M. Introduction to soil microbiology. 2.ed. New York : J. Wiley, 1977. 472p.

ALVAREZ, R.; DÍAZ， R.A.; BARBERO, N.; SANTANATOGLIA, O.J.; BLOTTA, L. Soil organic carbon, microbial biomass and $\mathrm{CO}_{2}-\mathrm{C}$ production from three tillage systems. Soil and Tillage Research, Amsterdam, v.33, n.1, p.17-28, jan. 1995a.

ALVAREZ, R.; TOGLIA, O.J.S.; DANIEL, P.E.; GARCIA, R. Respiration and specific activity of soil microbial biomass under conventional tillage and reduced tillage. Pesquisa Agropecuária Brasileira, Brasília, v.30, n.5, p.701-709, maio 1995b.

AZAM, F.; MULVANEY, R.L.; SIMMONS, F.W. Effects of ammonium and nitrate on mineralization of nitrogen from leguminous residues. Biology and Fertility of Soils, Berlim, v.20, n.1, p.49-52, 1995.

BARTHOLOMEW, W.V. Mineralization and immobilization of nitrogen in the decomposition of plant and animal residues. In: BARTHOLOMEW, W. V.; CLARK, F. E. (Eds.). Soil nitrogen. Madison : ASA, 1965. p.285-306. (Agronomy, 10). 
BREMER, E.; HOUTUM, W. van; VANKESSEL, C. Carbon dioxide evolution from wheat and lentil residues as affected by grinding, added nitrogen, and the absence of soil. Biology and Fertility of Soils, Berlim, v.11, n.3, p.222-227, 1991.

BREMNER, J.M.; KEENEY, D.R. Determination and isotoperatio analysis of different forms of nitrogen in soils. III. Exchangeable ammonium, nitrate and nitrite by extraction-distillation methods. Soil Science Society of America. Proceedings, Madison, v.30, n.5, p.577-582, Sept./Oct. 1966.

CARTER, M.R. Microbial biomass as an index for tillageinduced changes in soil biological properties. Soil and Tillage Research, Amsterdam, v.7, n.1/2, p.29-40, May 1986.

CHAN, K.Y.; ROBERTS, W.P.; HEENAN, D.P. Organic carbon and associated soil properties of a Red Earth after 10 years of rotation under different stubble and tillage practices. Australian Journal of Soil Research, Melbourne, v.30, n.1, p.71-83, 1992.

EMBRAPA. Centro Nacional de Pesquisa de Solos (Rio de Janeiro, RJ). Manual de métodos de análise de solo. 2.ed. rev. atual. Rio de Janeiro, 1997. 212p. (Embrapa-CNPS. Documentos, 1).

FOLLETT, R.F.; SCHIMEL, D.S. Effect of tillage practices on microbial biomass dynamics. Soil Science Society of America. Journal, Madison, v.53, n.4, p.1091-1096, July/Aug. 1989.

HENDRIX, P.F.; HAN, C.; GROFFMAN, P.M. Soil respiration in conventional and no-tillage agroecosystems under different winter cover crop rotations. Soil and Tillage Research, Amsterdam, v.12, n.2, p.135-148, Aug. 1988.

JENKINSON, D.S.; POWLSON, D.S. The effects of biocidal treatments on metabolism in soil. V. A method for measuring soil biomass. Soil Biology and Biochemistry, Oxford, v.8, n.3, p.209-213, 1976.
KNAPP, E.B.; ELLIOT, L.F.; CAMPBELL, G.S. Microbial respiration and growth during the decomposition of wheat straw. Soil Biology and Biochemistry, Oxford, v.15, n.3, p.319-323, 1983.

LEVI, M.P.; COWLING, E.B. Role of nitrogen in wood deterioration. VII. Physiological adaptation of wooddestroying and other fungi to substrates deficient in nitrogen. Phytopathology, St. Paul, v.59, p.460-468, Jan./Dec. 1969.

NEELY, C.L.; BEARE, M.H.; HARGROVE, W.L.P. Relationships between fungal and bacterial substrateinduced respiration, biomass and plant residue decomposition. Soil Biology and Biochemistry, Oxford, v.23, n.10, p.947-954, 1991.

PRIOR, S.A.; ROGERS, H.H.; RUNION, G.B.; TORBET, H.A.; REICOSKY, D.C. Carbon dioxide-enriched agroecosystems: influence of tillage on short-term soil carbon dioxide efflux. Journal of Environmental Quality, Madison, v.26, n.1, p.244-252, Jan./Feb. 1997

REINERTSEN, S.A.; ELLIOT, L.F.; COCHRAM, V.L.; CAMPBELL, G.S. Role of available carbon and nitrogen in determining the rate of wheat straw decomposition. Soil Biology and Biochemistry, Oxford, v.16, n.5, p.459-464, 1984.

SAMPAIO, E.V.S.B.; SALCEDO, I.H. Efeito da adição de nitrogênio e palha $\left(\mathrm{C}^{14}\right)$ na liberação de $\mathrm{CO}_{2}$ e formação de biomassa microbiana em Latossolo VermelhoAmarelo. Revista Brasileira de Ciência do Solo, Campinas, v.6, n.3. p.177-181, set./dez. 1982.

SANTRUCKOVA, H.; STRASKRABA, M. On the relationship between specific respiration activity and microbial biomass in soils. Soil Biology and Biochemistry, Oxford, v.23, n.6, p.525-532, 1991.

SWIFT, M.J.; HEAL, O.W.; ANDERSON, J.M. Decomposition in terrestrial ecosystems. Berkeley : Univ. of California Press, 1979. 372p. 